\title{
Gamma-rays from millisecond pulsar population in the Galactic Centre
}

\author{
Wlodek Bednarek* \\ Department of Astrophysics, University of Łódź \\ E-mail: bednareuni.lodz.p]

\section{T. Sobczak} \\ Department of Astrophysics, University of Łódź
}

\begin{abstract}
The central dense stellar cluster in the Galactic Centre has the mass of $\sim 4$ times larger than that of the central black hole. This cluster is expected to contain a large number of millisecond pulsars (MSPs) within the central parsec of the Galactic Centre provided by collapsing globular clusters. We propose that the $\mathrm{GeV} \gamma$-ray emission, observed from the Galactic Centre, is in fact a cumulative effect of the emission from several globular clusters captured by the Galactic Centre black hole. The millisecond pulsars accelerate leptons in their wind zones to energies of the order of a few tens of $\mathrm{TeV}$. We calculate the expected $\mathrm{TeV} \gamma$-ray emission produced by these leptons by the Inverse Compton Scattering process in the soft radiation field. It is shown that this emission can be responsible for the multi-TeV $\gamma$-rays observed by the Cherenkov telescopes from the Galactic Centre for reasonable densities of the soft radiation, diffusion models for the propagation of leptons, and their injection parameters (fluxes and spectral proprieties). If the energy conversion efficiency from the pulsars to the relativistic leptons is of the order of $10 \%$, then about a thousand of MSPs have to be present in the central cluster in the Galactic Centre.
\end{abstract}

Frontier Research in astrophysics,

26 - 31 May, 2014

Mondello (Palermo), Italy

* Speaker. 


\section{Introduction}

$\gamma$-ray emission was detected from the source 3EG J1746-2851 towards the Galactic Centre (GC) in the GeV energy range already by the EGRET telescope (Hartman et al. 1999, MayerHasselwander et al. 1998). The existence of this source was recently confirmed by the Fermi-LAT observations (Abdo et al. 2010a). Observations with various Cherenkov telescopes discovered also the $\gamma$-ray emission extending up to several TeV (Tsuchiya et al. 2004, Kosack et al. 2004, Aharonian et al. 2004, Albert et al. 2006). Although GC varies strongly at lower energies, the $\mathrm{GeV}-\mathrm{TeV}$ flux seems to be steady in the time period of several years. The $\gamma$-ray emission from GC shows two distinct components since an extrapolation of the multi-GeV spectrum does not meet the level of the $\mathrm{TeV}$ emission (Chernyakova et al. 2011). The $\mathrm{TeV}$ spectrum is well described by a power law (differential spectral index $2.1 \pm 0.04_{\text {stat }} \pm 0.10_{\text {syst }}$ ) with an exponential cut-off at $15.7 \pm 3.4_{\text {stat }} \pm 2.5_{\text {syst }} \mathrm{TeV}$ (Aharonian et al. 2009), extending up to a few tens of $\mathrm{TeV}$ (Aharonian et al. 2004). The $\mathrm{TeV}$ source is coincident with the position of the supermassive black hole Sgr A within an error circle of 13 arcsec. An upper limit on the source size of $1.3 \mathrm{arc}$ min, corresponding to $\sim 3 \mathrm{pc}$, was derived by Acero et al. (2010).

In this work, we consider the hypothesis in which the central stellar cluster in the GC is responsible for the high energy processes observed from the direction of Sgr $\mathrm{A}^{\star}$ (for details see Bednarek $\&$ Sobczak 2013). Following the proposition of Wang et al. (2005, see also Abazajian 2011), we assume that the $\mathrm{GeV} \gamma$-ray emission is due to the cumulative effect of several globular clusters captured by the central black hole. In fact, $\gamma$-ray emission with very similar spectral features has been recently detected by Fermi-LAT from globular clusters (Abdo et al. 2010b). We propose that the $\mathrm{TeV} \gamma$-rays from the direction of GC are produced by leptons with multi-TeV energies which Inverse Compton up-scatter the infrared and optical background in the central stellar cluster around the supermassive black hole identified with the Sgr A*.

\section{Central stellar cluster in the Galactic Centre}

The central region of the Galaxy contains a stellar cluster with the mass of $\sim 1.5 \times 10^{7} \mathrm{M}_{\odot}$ surrounding the central supermassive black hole with the mass $\sim 4 \times 10^{6} \mathrm{M}_{\odot}$. It is argued that such cluster could appear as a result of a merger of several globular clusters (GlCs) due to dynamical friction (Antonini et al. 2012, Capuzzo-Dolcetta et al. 2013). The central cluster has a uniform core with the radius of $R_{\mathrm{c}}=0.5 \mathrm{pc}$ and the stellar density profile outside the core described by the function $\rho \propto r^{-1.8}$ up to $\sim 30 \mathrm{pc}$. It is well known that globular clusters contain large populations of millisecond pulsars (MSPs). In fact, about half of observed MSPs were discovered within globular clusters (Harris 1996). It is estimated that some globular clusters may contain up to a few hundred MSPs. These expectations are consistent with the recent observations of the $\mathrm{GeV} \gamma$-ray emission from several GCs by the Fermi-LAT telescope (Abdo et al. 2009, Abdo et al. 2010b, Kong et al. 2010, Tam et al. 2011). If the above hypothesis is correct, then the central stellar cluster could contain up to a few tausend MSPs which might be responsible for the observed $\mathrm{GeV}$ emission from the Galactic Centre (see the model by Wang et al. 2005).

We propose that MSPs in the GC can accelerate leptons to $\sim 100 \mathrm{TeV}$ in their wind regions. In fact, efficient acceleration of particles to energies of this order is observed in the case of the 
winds around several classical pulsars. The acceleration in the winds of MSPs is expected to occur similarly since the processes in the inner magnetospheres of both groups of pulsars are expected to be the same (similar spectral features of the pulsed $\mathrm{GeV}$ emission). Note that a similar scenario has been recently considered in the models for the $\mathrm{TeV} \gamma$-ray emission from the globular clusters (e.g. Bednarek \& Sitarek 2007, Venter et al. 2009, Cheng et al. 2010).

For the basic parameters determining our model we adopt those generally consistent with the observational constraints on the magnetic field strength in the central region of the GC $(B>$ $50 \mu \mathrm{G}$, Crocker et al. 2010) and on the GC infrared and optical radiation field (energy densities $\sim 10^{4} \mathrm{eV} \mathrm{cm}^{-3}$, Mezger et al. 1996, Kusunose \& Takahara 2012). Leptons injected into such environment diffuse from the central cluster losing energy on the synchrotron and Inverse Compton (IC) radiation.

\section{Radiation from leptons}

In our model, MSPs are responsible for the acceleration of leptons in the central stellar cluster in GC. A MSP creates a strong pulsar winds with the power defined by the pulsar basic parameters,

$$
L_{\mathrm{p}} \approx 3.6 \times 10^{34} B_{8}^{2} / P_{3}^{4} \quad \mathrm{erg} \mathrm{s}^{-1},
$$

where $B_{\text {pul }}=3 \times 10^{8} B_{8} \mathrm{G}$ is the pulsar surface magnetic field strength, and $P_{\text {pul }}=3 P_{3} \mathrm{~ms}$ is the pulsar rotational period. The mean distance between MSPs within the central cluster in GC can be estimated as,

$$
D=3 \times 10^{17} R_{1} N_{3}^{-1 / 3} \mathrm{~cm},
$$

where $R_{\mathrm{c}}=1 R_{1} \mathrm{pc}$ is the core radius of the central cluster, and $N=10^{3} N_{3}$ is the number of MSPs within the central cluster. The MSPs move within the core of the central cluster with typical rotational velocities in the gravitational potential of the cluster of the order of,

$$
v_{\mathrm{r}}=\sqrt{2 G M / R} \approx 300\left(M_{7} / R_{1}\right)^{1 / 2} \mathrm{~km} \mathrm{~s}^{-1},
$$

where $G$ is the gravitational constant, $M=10^{7} M_{7} \mathrm{M}_{\odot}$ is the mass of the cluster within $R$.

The wind of MSP interacts with the cluster medium creating a bow shock with the radius, $R_{\mathrm{sh}}$, that can be determined from the comparison of the kinetic energy density of the medium (relative to pulsar) and the energy density of the pulsar wind,

$$
L_{\mathrm{p}} / 4 \pi c R_{\mathrm{sh}}^{2}=\rho v_{\mathrm{r}}^{2} / 2
$$

where $L_{\mathrm{p}}=10^{34} L_{34} \mathrm{erg} / \mathrm{s}$ is the energy loss rate of the pulsar, $c$ is the velocity of light, $R_{\mathrm{sh}}$ is the shock radius, $\rho=10^{3} \rho_{3} \mathrm{~cm}^{-3}$ is the density of matter in the central cluster, and $v=300 v_{3} \mathrm{~km} \mathrm{~s}^{-1}$ is the velocity of the pulsar. The distance of the shock from the pulsar is estimated from Eq. 3 and 4 ,

$$
R_{\mathrm{sh}}=1.9 \times 10^{14} L_{34}^{1 / 2} / \rho_{3}^{1 / 2} v_{3} \mathrm{~cm},
$$

i.e. it is much smaller than the characteristic distance between MSPs in the cluster core. We estimate the magnetic field strength at the shock from the pulsar site by scaling it from the pulsar 
surface. The dipole structure of the magnetic field is assumed within the light cylinder radius $\left(B(r) \propto r^{-3}\right)$ and its toroidal structure in the MSP wind region $\left(B(r) \propto r^{-1}\right)$. Then, the magnetic field strength at the shock is of the order of,

$$
B_{\mathrm{Sh}}=\eta B_{\mathrm{LC}} R_{\mathrm{LC}} / R_{\mathrm{sh}} \approx 7.5 \times 10^{-4} \eta_{-1} B_{8} \rho_{3}^{1 / 2} v_{3} /\left(L_{34}^{1 / 2} P_{3}^{2}\right) \quad \mathrm{G},
$$

where $B_{\mathrm{LC}}=B_{\mathrm{p}}\left(R_{\mathrm{NS}} / R_{\mathrm{LC}}\right)^{3}$ is the magnetic field strength at the light cylinder, $R_{\mathrm{NS}}=10^{6} \mathrm{~cm}$ is the neutron star radius, $\eta=0.1 \eta_{-1}$ is the magnetization parameter of the pulsar wind, and $R_{\mathrm{LC}}=c P / 2 \pi$ is the light cylinder radius.

We assume that leptons are accelerated at the shock reaching a power law spectrum up to the maximum energy defined by the escape from the shock region or by their radiation energy losses. After all, the Larmor radius of leptons accelerated at the pulsar wind shock,

$$
R_{\mathrm{L}}=c P / e B \approx 3 \times 10^{13} E_{\mathrm{TeV}} / B_{-4} \quad \mathrm{~cm}
$$

(where $E=1 E_{\mathrm{TeV}}$ is the lepton energy in $\mathrm{TeV}$ ), has to be smaller than the extent of the shock, $R_{\mathrm{sh}}$. This allows us to put the upper limit on the lepton energy of the order of,

$$
E_{\mathrm{L}} \approx 48 \eta_{-1} B_{8} / P_{3}^{2} \mathrm{TeV} \text {. }
$$

The energies of leptons can be also limitted by their synchrotron energy losses. Their maximum energies can be estimated by comparing the electron acceleration rate, $\dot{E}_{\mathrm{acc}}=\xi c E / R_{\mathrm{L}} \approx$ $10^{8} \xi_{-1} B_{-4} \mathrm{eV} \mathrm{s}^{-1}$ (where $\xi=0.1 \xi_{-1}$ is the acceleration coefficient), with the synchrotron energy loss rate, $\dot{E}_{\mathrm{syn}}=(4 / 3) c \sigma_{\mathrm{T}} U_{\mathrm{B}} \gamma^{2} \approx 35 B_{-4}^{2} E_{\mathrm{TeV}}^{2} \mathrm{eV} \mathrm{s}^{-1}$. This comparison gives $E_{\mathrm{syn}}^{\max } \approx 1.7 \times$ $10^{3}\left(\xi_{-1} / B_{-4}\right)^{1 / 2} \mathrm{TeV}$. The acceleration of leptons may be saturated also by their energy losses on the Inverse Compton process in the Klein-Nishina regime for the case of the infrared and optical photons. We roughly estimate lepton energy losses in the Klein-Nishina (KN) regime by applying the value of the transition from between the Thomson (T) and $\mathrm{KN}$ regime, $\dot{E}_{\mathrm{T} / \mathrm{KN}}=$ $(4 / 3) c \sigma_{\mathrm{T}} U_{\text {inf }} \gamma_{T / K N}^{2} \approx 6.7 \times 10^{3} \mathrm{eV} \mathrm{s}^{-1}$, where $\gamma_{T / K N}=m_{\mathrm{e}} c^{2} / \varepsilon_{\text {inf }} \sim 5 \times 10^{6}$ is the Lorentz factor of leptons corresponding to the transition from the $\mathrm{T}$ to $\mathrm{KN}$ regimes. The energy density of the infrared photons (with energies $\varepsilon_{\text {inf }} \sim 0.1 \mathrm{eV}$ ) is estimated as $U_{\text {inf }}=10^{4} \mathrm{eV} \mathrm{cm}^{-3}$ (Mezger et al. 1996), consistently with the observations of Davidson (1992). The optical background, which is less important here due to the more energetic photons, is estimated as $10^{(4-5)} \mathrm{eV} \mathrm{s}^{-1}$ (Mezger et al. 1996). Therefore, the IC energy losses in the KN regime can not additionally constrain the maximum energies of leptons accelerated to energies given by Eq. 8. We conclude that for likely parameters of the acceleration scenario (e.g. $\xi_{-1}=1$ for the relativistic pulsar wind and $B \sim 10^{-4}$ G) the limits due to energy losses of leptons are clearly less restrictive than the above limit due to the Larmor radii of particles. Therefore, we conclude that electrons can reach energies of the order of $\sim 100 \mathrm{TeV}$ for typical parameters of the MSPs within the central cluster (e.g. rotational period of $2 \mathrm{~ms}$ and surface magnetic field of $3 \times 10^{8} \mathrm{G}$, see e.g. Wang et al. 2005).

Leptons accelerated at the MSP wind shocks, with the power law spectrum extending up to energies of the order of a few tens of $\mathrm{TeV}$, diffuse outside the cluster interacting mainly with the infrared and optical radiation field of the central cluster. We consider three prescriptions for the diffusion model of leptons in the central cluster. In the first model, we assume that the magnetic field 
in the region between the shocks is highly turbulent. Therefore, the Bohm diffusion prescription seems to be appropriate. The diffusion coefficient is then given by,

$$
D_{\text {Bohm }} \approx 3 \times 10^{23}\left(E_{\mathrm{TeV}} / B_{-4}\right) \quad \mathrm{cm}^{2} \mathrm{~s}^{-1} .
$$

In the second diffusion model, we apply a prescription for the diffusion coefficient appropriate for the Kolmogorov spectrum of turbulence which is more characteristic for the medium in the galactic disk. In this case, the diffusion coefficient has the form,

$$
D_{\mathrm{Kol}} \approx 1.3 \times 10^{27}\left(E_{\mathrm{TeV}} / B_{-4}\right)^{1 / 3} \mathrm{~cm}^{2} \mathrm{~s}^{-1} .
$$

The diffusion coefficient has been normalized in the way described in Bednarek \& Protheroe (2002), i.e. to the case when the Larmor radius is equal to the extend of the size of the diffusion region assumed to be equal to $3 \mathrm{pc}$. In the third diffusion model we assume the Kolmogorov prescription for the diffusion coefficient but with its value one order of magnitude larger.

We calculate the IC $\gamma$-ray spectra produced by leptons in the model discussed above by applying the Monte Carlo code developed for the $\gamma$-ray production in GlCs (Bednarek \& Sitarek 2007). We follow the propagation of leptons in the magnetic and radiation field of the central stellar cluster up to the distance of $3 \mathrm{pc}$ from the Centre assuming that density of radiation is uniform within the core with the radius of $0.5 \mathrm{pc}$ and for larger distances drop according to $\propto r^{-1.8}$ (see CapuzzoDolcetta et al. 2013). These leptons are injected with the differential power law spectrum (spectral index 2.) and the cut-off at $50 \mathrm{TeV}$ (see Eq. 8) in the core of the cluster. It is found that $\gamma$-ray spectra depend weakly on the applied diffusion model of leptons within the central region of Galaxy. Only for the largest considered values of the diffusion coefficient, in the Kolmogorov model, the TeV flux (mainly around $\sim 100 \mathrm{GeV}$ ) starts to drop due to the escape of leptons from the cluster core.

\section{4. $\gamma$-ray spectrum from GC}

We propose a two component origin of the high energy $\gamma$-rays from the GC. The lower energy component, extending through the $\mathrm{GeV}$ energy range, is interpreted as due to the $\gamma$-ray emission from several globular clusters which create the central cluster as a result of the coalescence. This interpretation is supported by the recent discovery of $\mathrm{GeV} \gamma$-rays from several globular clusters (GlCs) (Abdo et al. 2010, Tam et al. 2011). In fact, the $\gamma$-ray spectra of some GlCs can be well described by a power law model with the exponential cut-off at a few GeV. However, the broken power law model also seems to work well for some GlCs especially for Ter 5, NGC 6652, M62 (see Fig. 2 in Abdo et al. 2010) or even a single power law model for NGC 6440 (Abdo et al. 2010). Moreover, Tam et al. (2011) explicitly state that discovered by them $\gamma$-ray emission from GlCs can be well described by a single power law model (see e.g. Fig. 5 for Liller 1 and Fig. 6 for NGC 6624 in that paper). As an example, we show in Fig. 1 the shape of the $\gamma$-ray spectra from two GlCs, Ter 5 and M62 (see solid lines marked by "I" and "II" GC MSP), observed by Fermi-LAT (Abdo et al. 2010b), after renormalization to the $\gamma$-ray flux observed from the GC. The observed level of GeV emission from the GC (e.g. Chernyakova et al. 2011) can be understood in terms of such interpretation provided that MSPs from several GlC (with typical GeV $\gamma$-ray luminosity of a few $10^{35} \mathrm{erg} \mathrm{s}^{-1}$, e.g. Ter 5, Lilier 1, or M62) are accumulated in the central stellar cluster. 


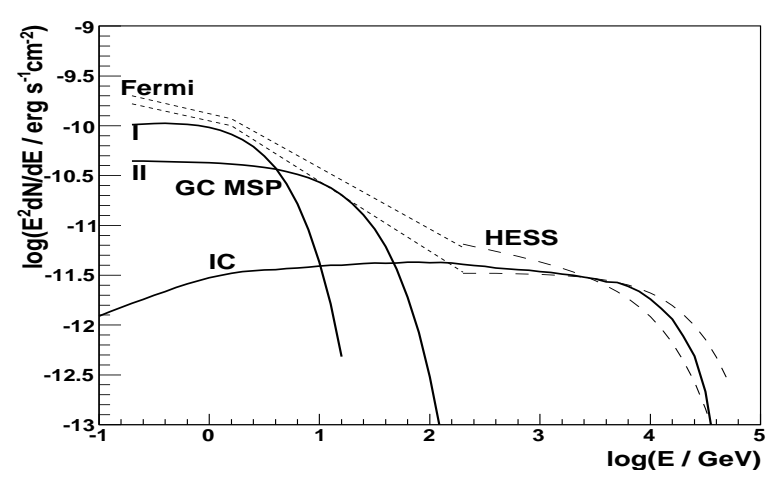

Figure 1: Spectral energy distribution of $\gamma$-rays from the Galactic Centre region measured by Fermi-LAT (dotted lines, Chernyakova et al. 2011) and the HESS Collaboration (dashed curves, Aharonian et al. 2009). The GeV $\gamma$-rays are interpreted as the contribution from the population of the millisecond pulsars present in the central region of Galaxy as a result of a merger of several globular clusters. As illustration, we show $\gamma$-ray spectra of two GICs: Ter 5, solid curves marked by I, and M 62, marked by II (see Abdo et al. 2010b), after renormalization to the $\mathrm{GeV}$ flux from the GC. The $\mathrm{TeV} \gamma$-rays are produced by IC of leptons accelerated in the MSP wind regions. The calculations have been done for: $B=10^{-4} \mathrm{G}, L=10^{7} \mathrm{~L}_{\odot}, u_{\text {inf }}=10^{4} \mathrm{eV}$ $\mathrm{cm}^{-3}, R=3 \mathrm{pc}$, and the Bohm diffusion model with Eq. 10.

The second, higher energy component, in the $\gamma$-ray spectrum observed from the GC by Cherenkov telescopes is interpreted as due to the IC scattering of soft infrared and optical background radiation by relativistic leptons accelerated by this same population of MSPs. As an example, we compare the IC $\gamma$-ray spectrum produced by these leptons with a power law spectrum, with the spectral index equal to 2 extending up to $50 \mathrm{TeV}$ with the HESS TeV measurements. The observed level of $\mathrm{TeV}$ $\gamma$-ray emission can be understood in terms of this interpretation provided that the product of the energy conversion efficiency from the MSPs to relativistic leptons, $\chi$, times the number of MSPs, $N_{\mathrm{MSP}}$, is of the order of $\chi \cdot N_{\mathrm{MSP}} \sim 110$, if the typical parameters of the MSPs are $B_{\mathrm{MSP}}=3 \times 10^{8}$ $\mathrm{G}$ and $P_{\mathrm{MSP}}=3 \mathrm{~ms}$. If the acceleration of leptons in winds of MSPs occurs as observed around classical pulsars, then about a thousand of MSPs should be present in the central stellar cluster around Sgr $\mathrm{A}^{\star}$. We find that the synchrotron X-ray emission from leptons accelerated by pulsars is consistent with the low state of the X-ray emission observed from central parsec around Sgr $\mathrm{A}^{\star}$ by Chandra in the energy range 2-10 keV (Baganoff et al. 2003), provided that the average magnetic field strength in this region is not significantly larger than $\sim 100 \mu \mathrm{G}$.

In the model discussed above we consider also the extend of the TeV $\gamma$-ray source. This diffusion distance can be estimated from $R_{\mathrm{dif}}=\sqrt{2 D \tau_{\mathrm{IC}}^{\mathrm{T} / \mathrm{KN}}}$, where $D$ is the diffusion coefficient given by Eq. (10) or Eq. (11), and $\tau_{\mathrm{IC}}^{\mathrm{T} / \mathrm{KN}}=E_{\mathrm{e}} / \dot{E}_{\mathrm{T} / \mathrm{KN}}$ is the energy loss time scale of electrons in the $\mathrm{KN}$ regime, and the energy loss rate in the Klein-Nishina regime, $\dot{E}_{\mathrm{T} / \mathrm{KN}}$, is given above. The largest energy losses of electrons are expected due to their scattering of the infrared radiation. For the parameters given above, the diffusion distance is estimated on $R_{\mathrm{dif}}^{\mathrm{Bohm}} \approx 9.5 \times 10^{15} E / B_{-4}^{1 / 2} \mathrm{~cm}$ in the case of Bohm diffusion and $R_{\mathrm{dif}}^{\mathrm{Kol}} \approx 6.2 \times 10^{17} E^{2 / 3} / B_{-4}^{1 / 6} \mathrm{~cm}$ in the case of Kolmogorov diffusion. For the electrons with energies of $50 \mathrm{TeV}$, these diffusion distances are equal to $\sim 4.7 \times 10^{17} \mathrm{~cm}$ and $\sim 8.5 \times 10^{18} \mathrm{~cm}$, respectively for the Bohm and Kolmogorov diffusion coefficients. These diffusion distances are smaller than (or comparable to) the upper limit on the extend of the $\mathrm{TeV} \gamma$-ray source 
at the Galactic Centre estimated on 2.9 pc by the HESS Collaboration (Acero et al. 2010).

\section{Conclusion}

The $\mathrm{GeV} \gamma$-ray emission from the central parsec around $\mathrm{Sgr} \mathrm{A}^{\star}$ can be produced in the central cluster which is due to the capture of several globular clusters by the central black hole (Antonini et al. 2012). We explain the steady $\mathrm{TeV} \gamma$-ray emission from this region as being produced by relativistic leptons, accelerated in the MSP wind regions, which up-scatter dense infrared and optical background radiation. The product of the conversion of the rotational energy lost by the MSPs to relativistic leptons, $\chi$, and the number of MSPs in the central cluster, $N_{\mathrm{MSP}}$, has to be of the order of $\sim 110$, provided that a typical pulsar magnetic field is $3 \times 10^{8} \mathrm{G}$ and the rotational period is 3 ms (see Wang et al. 2005). The value of $\chi \sim 10 \%$ postulates the existence of about $10^{3}$ MSPs in the central cluster around Sgr $A^{\star}$. We show that the extend of the $\mathrm{TeV} \gamma$-ray source expected in our model is consistent with the upper limit on the source size derived by the HESS Collaboration (Acero et al. 2010). Our model excludes any significant variability of the $\mathrm{GeV}-\mathrm{TeV} \gamma$ ray spectrum from the central region around $\mathrm{Sgr} \mathrm{A}^{\star}$ below the presently observed emission level. Therefore, an observation of $\mathrm{GeV}-\mathrm{TeV} \gamma$-ray emission on the level clearly below the presently observed one would be inconsistent with this model.

\section{Acknowledgments}

This work is supported by the grant through the Polish Narodowe Centrum Nauki No. 2011/01/B/ST9/0041).

\section{References}

[1] Abazajian, K.N. 2011 JCAP 03, 010

[2] Abdo, A.A. et al. 2009 Science 325, 845

[3] Abdo, A.A. et al. 2010a ApJS 188405

[4] Abdo, A.A. et al. 2010b A\&A 524, A75

[5] Acero, F. et al. 2010 MNRAS 402, 1877

[6] Aharonian, F. et al. 2004 A\&A 425, 13

[7] Aharonian, F. et al. 2009 A\&A 503, 817

[8] Albert, J. et al. 2006 ApJ 638, 101

[9] Antonini, F., Capuzzo-Dolcetta, R., Mastrobuon-Battisti, A., Merritt, D. 2012 ApJ 750, 111

[10] Baganoff, F.K. et al. 2003 ApJ 591, 891

[11] Bednarek, W, Protheore, R.J. 2002 APh 16, 397

[12] Bednarek, W., Sitarek, J. 2007 MNRAS 337, 920

[13] Bednarek, W., Sobczak, T. 2013 MNRAS 435, L14

[14] Capuzzo-Dolcetta, R, Arca-Sedda, M., Spera, M. 2013, arXiv:1302.2509 
[15] Cheng, K.S. et al. 2010 ApJ 723, 1219

[16] Chernyakova, M. et al. 2011 ApJ 726, 60

[17] Crocker, R.M. et al. 2010 Nature 463, 65

[18] Davidson, J.A. 1992 ApJ 387, 189

[19] Harris, W.E. 1996 AJ 112, 1487

[20] Kong, A.K. et al. 2010 ApJ 712, 36

[21] Kosack, K. et al. 2004 ApJ 608, 97

[22] Kusunose, K., Takahara, F. 2012 ApJ 748, 34

[23] Mayer-Hasselwander, H.A., et al. 1998 A\&A 335, 161

[24] Mezger, P.G., Duschl, W.J., Zylka, R. 1996 A\&AR, 7, 289

[25] Tam, P.H.T. et al. 2011 ApJ 729, 90

[26] Tsuchiya, K. et al. 2004 ApJ 606, 115

[27] Venter, C. et al. 2009 ApJ 696, L52

[28] Wang, W., Jiang, Z.J., Cheng, K.S. 2005 MNRAS 358, 263

\section{DISCUSSION}

JEAMS H. BEALL: What is the time scale for collapse of the globular clusters onto the GC ?

WLODEK BEDNAREK: The time scale for collapse of the globular clusters onto the GC is $\sim 10$ Gyrs (see simulations by Antonini et al. 2012).

JEAMS H. BEALL: How sensitive is your calculation to the position of the pulsar's shock?

WLODEK BEDNAREK: The maximum energies of leptons, accelerated in the pulsar winds, are dependent on the maximum available potential drop which scales with the distance from the pulsar and the value of the local magnetic field strength in the wind. Since the maximum potential in the pulsar wind is independent on the distance from the pulsar (due to the toroidal structure of the magnetic field), the maximum energies of leptons (and so the results of calculations) do not depend on the position of the shock in the pulsar wind. 